\title{
A case of Gitelman syndrome with normomagnesemia
} Do detailed history and basic laboratory tests provide correct diagnosis?

\author{
Yumi Harano, Kazufumi Honda, Yurika Akiyama, Hiroko Arioka
}

Department of General Internal Medicine, St. Luke's International Medical Center, Tokyo, Japan

\section{Introduction}

Gitelman syndrome (GS) is autosomal recessive disorder, characterized by hypokalemia, hypomagnesemia, metabolic alkalosis and low urinary calcium excretion. We report a case with final diagnosis of GS using the DNA analysis, presented with severe hypokalemia but normomagnesemia. 